\title{
Educación ambiental para el manejo apropiado de agrotóxicos en comunidades rurales de Manabí.
}

Environmental education for the proper management of agrotoxic in rural communities of Manabí.

Educação ambiental para o manejo adequado de agrotóxicos nas comunidades rurais de Manabí.

María Isabel Delgado Moreira/ ESPAM Manuel Félix López, Ecuador/ mariai.delgado@espam.edu.ec

María Virginia Moreira Macías / ESPAM Manuel Félix López, Ecuador/ mariavi.moreira@espam.edu.ec

Diana Beatriz Vidal Zambrano/ ESPAM Manuel Félix López, Ecuador/ diana.vidal@espam.edu.ec

Yomaly Melissa Andrade Macías/ ESPAM Manuel Félix López, Ecuador/yomaly.andrade@espam.edu.ec

Enrique Richard/ Universidad Andina Simón Bolívar, Ecuador/ chelonos@gmail.com

Recibido: 31/7/2020 Aceptado: 9/12/2020 Publicado: 30/12/2020

\section{RESUMEN}

Los agrotóxicos se utilizan mundialmente para aumentar la producción de alimentos. El objetivo de esta investigación fue aplicar una estrategia de educación ambiental para el manejo adecuado de agrotóxicos en la Escuela Miguel Solórzano. La metodología empleada tuvo un enfoque cualitativo. La investigación se desarrolló en tres fases: diagnóstico, diseño e implementación de la estrategia y evaluación de los resultados. Acorde a las características de la población, se trabajó con dos grupos: niños y adultos. La evaluación reveló que el desconocimiento sobre los agrotóxicos se redujo en un $23 \%$ y la comprensión sobre el daño que estos productos pueden causar a la salud se incrementó en un $40 \%$; el grado de preocupación por el efecto de los plaguicidas aumentó en un $44 \%$, por lo que el porcentaje de uso de equipos de protección aumentó en un 44\%. En la disposición final de envases de plaguicidas, la entrega de envases vacíos al centro de acopio pasó de $0 \%$ a $80 \%$. Luego de aplicar la estrategia de educación ambiental se evidencia un cambio de perspectiva acerca del manejo de agrotóxicos, lo cual muestra la pertinencia de las intervenciones de este tipo en comunidades rurales.

Palabras clave: ambiente, capacitación, contaminación, estrategia de educación

\footnotetext{
* Mención especial del I Talller Nacional Estudiantil sobre Medio Ambiente - Ecuador 2020
} 


\section{ABSTRACT}

Agrotoxics are used worldwide to increase food production. The objective of this research was to apply a strategy through environmental education for the proper management of agrotoxics at Miguel Solórzano School. A qualitative methodology was used. The research was developed in three phases: the diagnosis, design and implementation of the strategy and evaluation of results. According to the characteristics of the population, we worked with two groups: children and adults. The evaluation of the strategy revealed that ignorance about pesticides decreased by $23 \%$ and understanding about the damage that these products can cause to health increased by $40 \%$; the degree of concern about the effect of agrotoxics increased by $44 \%$, so that the percentage of use of protective equipment increased by $44 \%$. In the final disposal of agrotoxics containers, the delivery of empty containers to the collection center went from $0 \%$ to $80 \%$. After applying the environmental education strategy, there is evidence of a change in perspective regarding the management of agrotoxics.

Keywords: education strategy, environment, pollution, training

\section{RESUMO}

Os agrotóxicos são usados em todo o mundo para aumentar a produção de alimentos. 0 objetivo desta pesquisa foi aplicar uma estratégia por meio da educação ambiental para o manejo adequado de agrotóxicos na escola Miguel Solórzano. Utilizou-se a metodologia qualitativa e, por meio de métodos empíricos, foi desenhada a estratégia de educação ambiental aplicada, foram estabelecidas três fases que incluíram o diagnóstico, a a plicação da estratégia e sua avaliação. De acordo com as características da população, trabalhamos com dois grupos: crianças e adultos. A avaliação da estratégia revelou que o desconhecimento sobre os agrotóxicos diminuiu $23 \%$ e o conhecimento sobre os danos que esses produtos podem causar à saúde aumentou em 40 \%; o grau de preocupação com o efeito dos agrotóxicos aumentou $44 \%$, de modo que o percentual de uso de equipamentos de proteção aumentou $44 \%$. Na destinação final das embalagens de agrotóxicos, a entrega das embalagens vazias na central de coleta passou de 0\% para $80 \%$. Após a aplicação da estratégia de educação ambiental, constatou-se uma mudança de perspectiva quanto ao manejo de agrotóxicos.

Palavras chave: estratégia educacional, meio ambiente, poluição, treinamento

\section{INTRODUCCIÓN}

Los agrotóxicos son sustancias químicas que se utilizan para influir en el proceso de producción de alimentos con la finalidad de aumentarla. En la actualidad existen más de 1000 ingredientes activos que se distribuyen entre insecticidas, herbicidas y fungicidas. Contradictoriamente, aunque son considerados a nivel mundial como uno de los principales factores involucrados en la contaminación ambiental, constituyen también una de las pocas sustancias tóxicas 
también una de las pocas sustancias tóxicas que están disponibles deliberadamente para su uso en el control de plagas y vectores de enfermedades. Por tal razón, la formulación de nuevos y potentes agrotóxicos está aumentando debido a la aparición de plagas resistentes o controles sanitarios; además de la creciente necesidad de producción de alimentos vinculada al crecimiento de la población mundial (Pacheco y Barbona, 2017).

En la última década, América Latina se ha convertido en una de las principales regiones asociadas al consumo mundial de dichos productos. En esta región, los habitantes de las zonas rurales corren el riesgo de exponerse a ellos. Si bien la mayoría de los agrotóxicos identificados como fuertemente dañinos han sido prohibidos en varias regiones del mundo, a raíz de la posibilidad de generar un deterioro crónico en la salud de las personas, aún se utilizan: atrazina, paraquat, glifosato herbicida (GBH), clorpirifos (CPF), mancozeb e imidacloprid (Rossetti et al., 2020).

En tal sentido, los agrotóxicos provocan numerosos conflictos en la salud humana, animal y vegetal, pues estos son propagados en el ambiente y su modo de acción involucra destruir los sistemas o enzimas de plagas que pueden ser similares o idénticos a los sistemas o enzimas de los seres humanos; por ejemplo, algunos pesticidas son inmunomoduladores que pueden incrementar enfermedades infecciosas en los seres vivos, o son disruptores endócrinos de humanos con efectos potenciales sobre la inmunidad (Rohr et al., 2019).

En Ecuador, el $71 \%$ de las intoxicaciones se deben a organofosforados y carbamatos, los cuales causan la muerte en un $4 \%$ de los casos. En este país, el $49.20 \%$ de quienes aplican agroquímicos (agrotóxicos y fertilizantes) son productores o agricultores, seguidos por los jornaleros o peones en un $34.16 \%$, y apenas un $3.91 \%$ son técnicos especializados; en el $12.70 \%$ restante, la aplicación se realiza por un miembro de la familia (Instituto Nacional de Estadística y Censos [INEC], 2016). Sin embargo, son precisamente los trabajadores agrícolas quienes presentan mayor vulnerabilidad a los riesgos producidos por agrotóxicos, pues manipulan estas sustancias sin las medidas de higiene y seguridad adecuadas (Naranjo, 2017).

Por otra parte, Hyland \& Laribi (2017) señalan que los niños de comunidades rurales son los más expuestos a estos productos, a través de diversos patrones que incluyen el almacenamiento de dichas sustancias en sus hogares. Por lo que, tal como indican Decnovk et al. (2019), los efectos por el uso de agrotóxicos reflejados en la salud de los usuarios directos, de la población expuesta indirectamente y los daños al ambiente, han planteado la urgente necesidad de formular programas y medidas preventivas que garanticen el manejo seguro de ellas.

A nivel nacional el uso de agrotóxicos sigue en aumento, pues entre 2006 y 2010 se cuadruplicaron las toneladas de pesticidas por cada mil hectáreas, es decir, que para el 2010 la correlación de kilogramos de plaguicidas por individuo fue de $6.35 \mathrm{~kg}$ (INEC, 2016; Ministerio de Agricultura, Ganadería, Acuacultura y Pesca, 2016).

Desde finales de 2017, la Asamblea General de las Naciones Unidas adoptó por consenso la resolución: Educación para el Desarrollo 
Sostenible en el marco de la Agenda 2030, donde se resalta que la educación ambiental (educación para el desarrollo sostenible), es reconocida como una herramienta principal y transversal, con importancia crítica para promover el desarrollo sostenible y aumentar la capacidad de las poblaciones para abordar cuestiones ambientales y de desarrollo. En tal sentido, la Estrategia Nacional de Educación Ambiental para el Desarrollo Sostenible 2017-2030, de Ecuador, especifica que: «La Educación Ambiental constituye una estrategia para la difusión, sensibilización y capacitación de la ciudadanía; de esta manera apoya y fortalece el concepto de desarrollo sostenible, considerando las necesidades de las futuras generaciones» (Ministerio del Ambiente del Ecuador, 2018, p. 4).

Por su parte, Bravo et al. (2020) indican que el uso de agrotóxicos en la actividad agrícola es predominante en la provincia ecuatoriana de Manabí. Asimismo, en las comunidades rurales, donde básicamente se realiza la producción de alimentos, las intervenciones de educación ambiental son escasas; por tanto, la presente investigación tuvo como objetivo aplicar una estrategia de educación ambiental para el manejo adecuado de agrotóxicos en la Escuela Miguel Solórzano. El aporte de la presente investigación es contribuir al mejoramiento de la calidad de vida de los moradores de comunidades rurales en Manabí, e impulsar futuros trabajos sobre el tema en estudio.

\section{MATERIALES Y MÉTODOS}

El trabajo se realizó en la Escuela Miguel Solórzano del sitio Bravos Grandes, en las coordenadas 594903.10 E y 9930968.93 S (parroquia San Antonio, Manabí, Ecuador). En esta zona rural, la actividad agrícola es predominante, destacándose los cultivos de maíz, plátano, yuca y maracuyá (figura 1); la aplicación de agrotóxicos se realiza durante la época lluviosa en la fase inicial (siembra) y la fase intermedia (floración).

Figura 1. Ubicación satelital Escuela Miguel Solórzano.

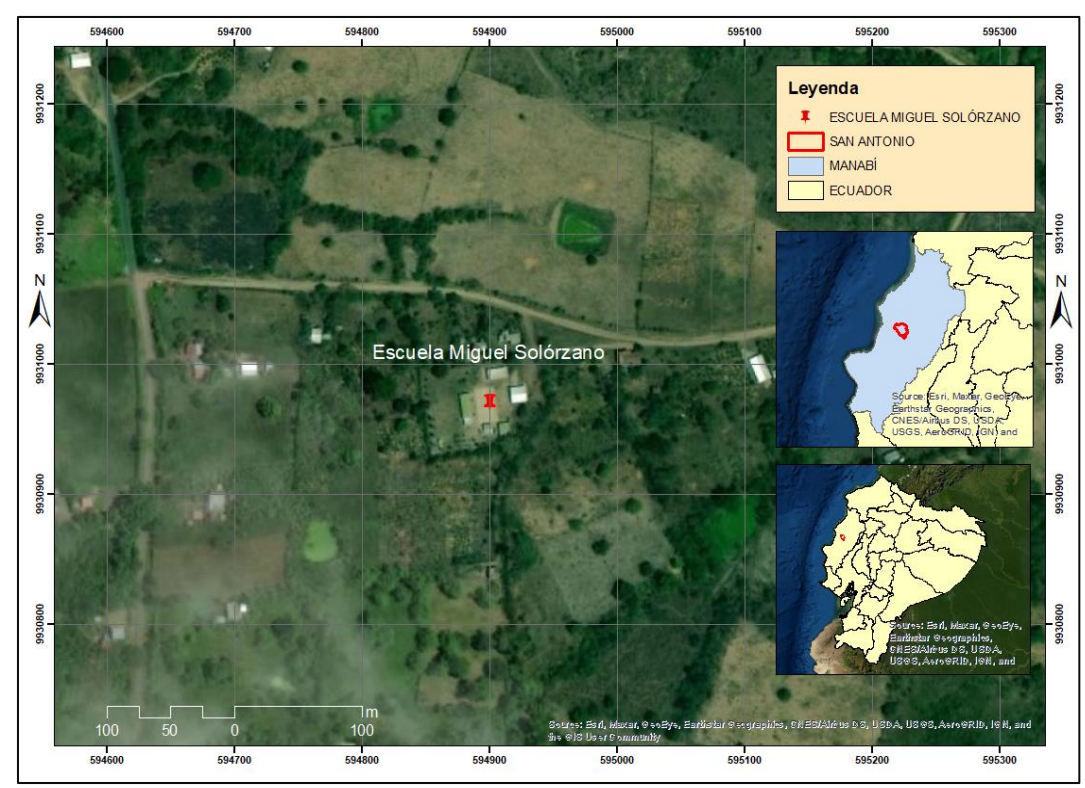

Fuente: Elaboración propia. 
La metodología empleada fue cualitativa y mediante los métodos teóricos y empíricos se estableció la estrategia de educación ambiental aplicada en la Escuela Miguel Solórzano (principal institución de la comunidad Bravos Grandes). Como parte de la misma se realizaron charlas los días martes y jueves durante los meses de diciembre de 2019, enero y febrero de 2020. A continuación, se detallan las fases de la investigación:

\section{Diagnóstico e inventario del uso de agrotóxicos en el sitio Bravos Grandes}

Para la recolección de los datos se emplearon técnicas como la entrevista grupal semiestructurada y la observación estructurada directa grupal, a partir de las características de la población, a las reuniones grupales que se realizaban por notificación del líder comunitario.

La muestra (n) se determinó utilizando la ecuación propuesta por Herrera (2011):

$$
n=\frac{N * Z_{\alpha}^{2} * p * q}{d^{2} *(N-1)+Z_{a}^{2} * p * q}
$$

Donde:

$\mathrm{N}=$ Total de la población

$Z \alpha=1.96$ al cuadrado (si la seguridad es del 95\%) $p=$ proporción esperada (en este caso $5 \%=0.05$ )

$q=1-p($ en este caso $1-0.05=0.95)$

$\mathrm{d}=$ precisión $(5 \%)$

\section{Ejecución de la estrategia de educación ambiental para un manejo adecuado de agrotóxicos, sus envases y residuos en el sitio Bravos Grandes}

Acorde a las características de la población, se trabajó con dos grupos: niños y adultos. La estrategia de educación ambiental se diseñó teniendo en cuenta las edades de los grupos y se adaptaron las actividades empleadas por Shattuck (2019), las que se detallan a continuación:

\section{Programa para niños}

- Obra: El burro y sus envases

Síntesis: La obra representa la intoxicación de Juanito cuando en su familia se usan envases vacíos de agrotóxicos para consumir alimentos. Además, se incluye una explicación médica de lo sucedido y de cómo los niños pueden ser los más afectados por estas sustancias. Se incluyeron reflexiones de los niños en la obra y, debido al afecto que los alumnos mostraron por los títeres, estos se entregaron a los docentes para que sean parte del material didáctico de la escuela.

- Canción: Cuidado con los agrotóxicos

Hay un planeta por recuperar, una mentalidad hay que cambiar, para que producir sin preocupación, cuidar el ambiente es nuestra misión.

Tenemos problemas de actitud, debemos cuidar nuestra salud, los desechos tóxicos vendrán, a contaminar el agua y nuestro hogar.

Si reutilizamos está bien, pero deberíamos de aprender, que con los químicos no hay que jugar, sus desechos nos pueden matar. Niños, si hago un vaso con un envase de veneno ¿estará bien?

(CORO) 
Si dice veneno me puede enfermar, si dice cuidado no debo tocar, si dice peligro me debo cuidar, o terminaré en el hospital.

Los envases hay que depositar, en los centros de acopio de nuestra ciudad y debemos siempre recordar, que a los ríos no hay que tirar.

Niños, y si me baño con estos envases ¿me puedo enfermar? (CORO)

Si dice veneno me puede enfermar, si dice cuidado no debo tocar, si dice peligro me debo cuidar, o terminaré en el hospital,

Los envases hay que depositar, en los centros de acopio de nuestra ciudad y debemos siempre recordar que a los ríos no hay que tirar.

\section{Programa para adultos}

- Presentación: uso del Equipo de Protección Personal (EPP)

En la presentación realizada se hizo énfasis en el uso de EPP para el manejo adecuado de agrotóxicos, por lo que se detalló la funcionalidad de cada unoz de ellos. También se proyectaron dos videos explicativos sobre cómo debe utilizarse el EPP y el orden en el que deben colocarse las prendas. Además, se detalló la manera en la que debe efectuarse la disposición final de los envases vacíos. Se dio a conocer que en la parroquia San Antonio existe un centro de acopio de estos, mediante el cual se pueden gestionar de manera adecuada esos desechos.

\section{Evaluación de la estrategia aplicada}

El cuestionario de diagnóstico se aplicó nuevamente luego de ejecutarse la estrategia de educación ambiental, los datos se tabularon y contrastaron mediante gráficos con el empleo del software Microsoft Excel 2016.

\section{RESULTADOS Y DISCUSIÓN}

Como evaluación, luego de aplicar las actividades de El burro y sus envases y Cuidado con los agrotóxicos, se tomaron las reflexiones de los niños sobre el uso de esas sustancias en su comunidad y se evidenció un cambio de conducta hacia las mismas y su manejo. Con respecto a los adultos, se aplicó la encuesta a una muestra de 57 personas (población total: 252 habitantes).

De acuerdo a la segunda aplicación del cuestionario, se logró reducir de 33\% a 10\% el desconocimiento sobre los agrotóxicos (figura 2), lo cual reafirma los criterios de Mantecón (2015) al decir que la educación ambiental permite informar a las personas sobre los graves efectos que provocan estos productos y es factible explicar la situación en la que está sumida una localidad. 
Figura 2. Incremento en el conocimiento sobre agrotóxicos.

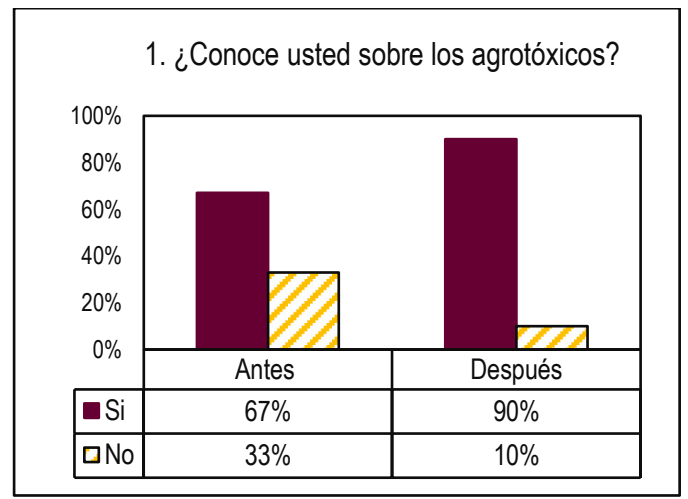

Fuente: Elaboración propia.

En cuanto a la frecuencia de uso, después de aplicarse la estrategia de educación ambiental, el $60 \%$ de los participantes respondió que siempre utiliza agrotóxicos, el 30\% (5\% menos) afirma que a veces lo utiliza y el $10 \%$ que nunca lo hace (figura 3 ). Estos resultados se relacionan con el estudio realizado por García (2012) en el cual se detalla que un $67 \%$ de los agricultores utilizan agrotóxicos dos veces al año, hecho vinculado a su empleo en la producción de alimentos que se obtienen en la comunidad de estudio.

Figura 3. Frecuencia de uso de agrotóxicos.

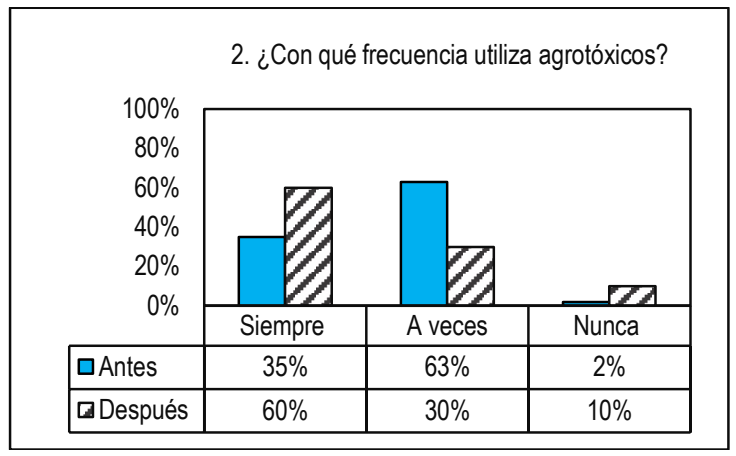

Fuente: Elaboración propia.

Antes de aplicar la estrategia, el $40 \%$ de los encuestados desconocía los daños que los agrotóxicos pueden causar a la salud. Después de ejecutar la estrategia el $80 \%$ aseguró que sí pueden provocar efectos nocivos (figura 4). Tal como sugieren Elahi et al. (2019), la producción social de riqueza va acompañada sistemáticamente por la producción social de riesgos, por lo cual, la sociedad del riesgo comienza donde falla la seguridad en los sistemas de normas sociales, en relación con los peligros desatados por los riesgos sociales, ecológicos, políticos e individuales. 
Figura 4. Incremento en el conocimiento sobre agrotóxicos.

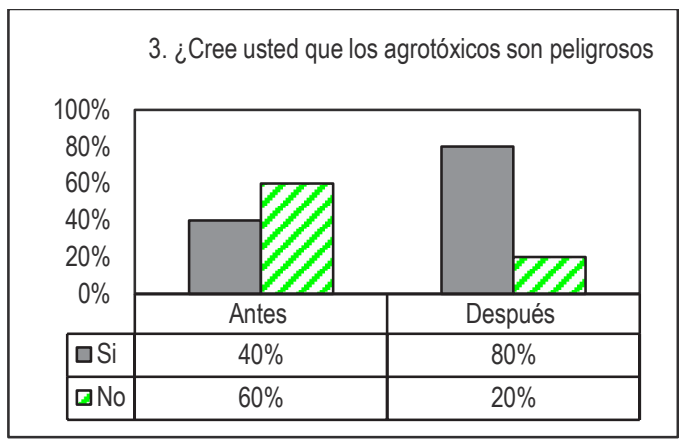

Fuente: Elaboración propia.

De manera similar, después de la aplicación de la estrategia, se incrementó en un 17\% (de 63\% a $80 \%$ ) el conocimiento de los síntomas que se presentan ante la exposición continua a agrotóxicos (figura 5). Según el estudio realizado por Yanggen et al. (2003), investigadores del Centro Internacional de la
Papa, es preocupante que entre las personas que reconocen los síntomas o signos de intoxicación por agrotóxicos, el mayor porcentaje indica haberlos experimentado él mismo, por lo que se destaca la importancia de fomentar la percepción sobre los efectos de los agrotóxicos en la salud.

Figura 5. Concepciones sobre la exposición continua a agrotóxicos.

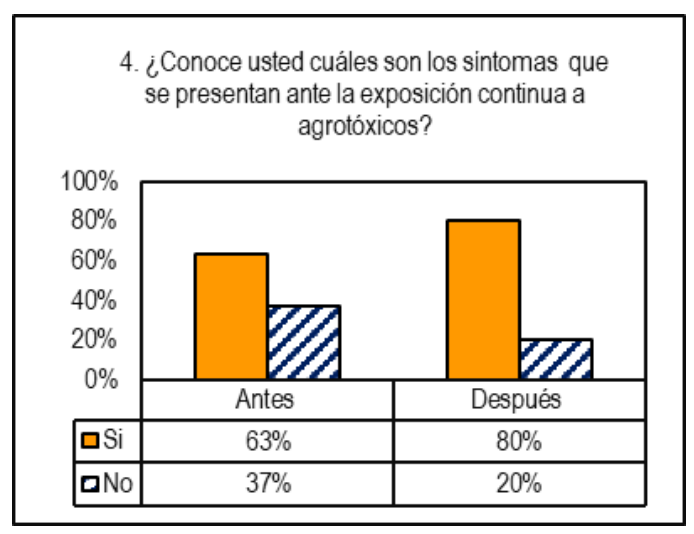

Fuente: Elaboración propia.

Después de aplicarse la estrategia, se evidenció un cambio de percepción en cuanto a las enfermedades que pueden provocar los agrotóxicos, pues del $9 \%$ de las personas que consideraban que los agrotóxicos pueden provocar cáncer, aumentó a un $40 \%$. Con respecto a las malformaciones congénitas, el $7 \%$ consideraba que estos productos tienen incidencia en esa enfermedad, pero en la actualidad el $10 \%$ lo afirma. En cuanto a las demás enfermedades consideradas en esta pregunta, las cifras bajaron considerablemente debido a que la mayoría de las personas encuestadas mencionaron la relación de esas sustancias con el cáncer (figura 6). 
Tal como mencionan Ferreira et al. (2015), aunque algunos agrotóxicos pueden clasificarse como moderadamente o ligeramente tóxicos en función de sus efectos agudos, no se pueden perder de vista los efectos crónicos que pueden ocurrir meses, años o incluso décadas después de la exposición, manifestándose en diversas enfermedades, tales como el cáncer, las malformaciones congénitas, los trastornos endocrinos, neurológicos y mentales. Además, Bhandari (2014) recalca que el empleo de dichas sustancias entraña una marcada conciencia del riesgo ambiental y de salud, donde las enfermedades prevalentes son las respiratorias, metabólicas, cardiovasculares, de piel y neoplásicas. Elahi et al. (2019) encontraron que la ocurrencia de irritación ocular, mareo, tos y náusea se relaciona significativamente con la aplicación de agrotóxicos. Por su parte, García (2012) encontró que todos los agricultores, después de usar agrotóxicos con frecuencia, mencionan presentar dolores de cabeza o estómago.

Figura 6. Enfermedades asociadas con el uso de agrotóxicos.

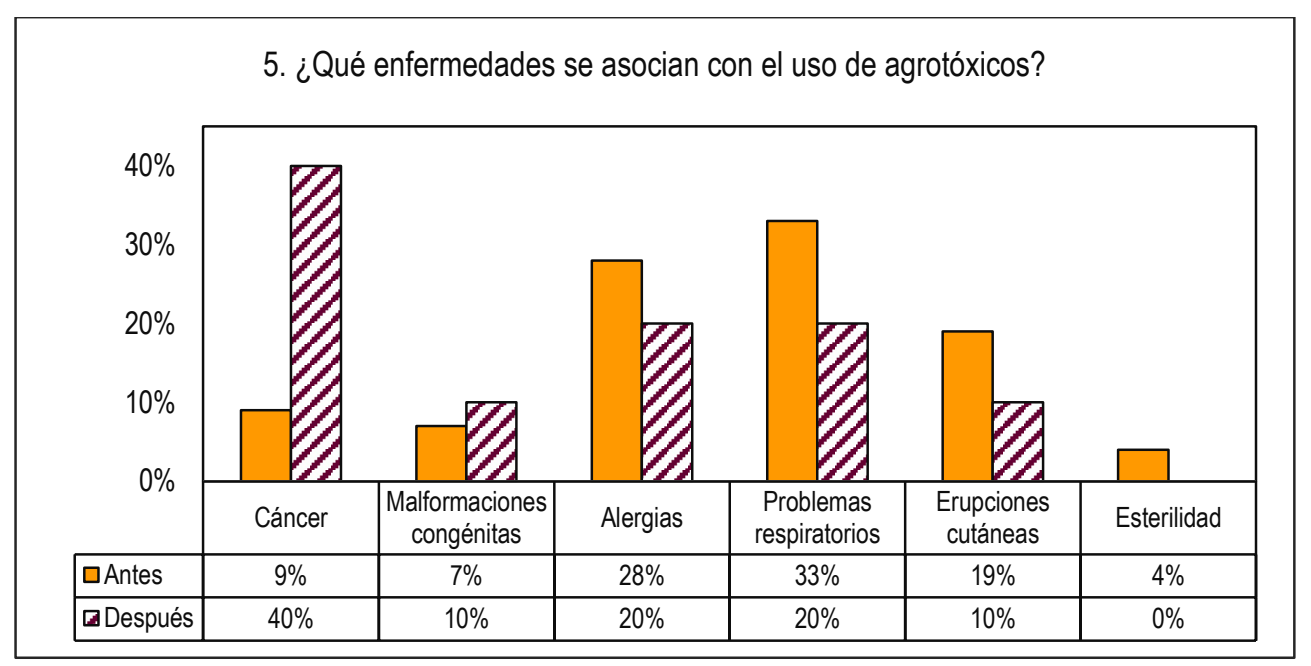

Fuente: Elaboración propia.

Antes de aplicar la estrategia solo un 16\% de los encuestados presentaba un grado de preocupación muy alto frente al efecto de los agrotóxicos; sin embargo, después de ejecutar la estrategia este porcentaje subió al 60\%. No obstante, un $10 \%$ de los encuestados afirmó no tener ninguna preocupación sobre dicho efecto a pesar de que los agricultores mencionaron que se han dado varios casos de intoxicaciones (figura 7). Aunque ya existe evidencia del vínculo de ciertos agrotóxicos con diversas enfermedades, resulta prácticamente imposible aseverar los padecimientos específicos que se deban al uso o contacto con estas sustancias (Hidalgo et al., 2016). 
Figura 7. Grado de preocupación referente a los efectos del uso de agrotóxicos.

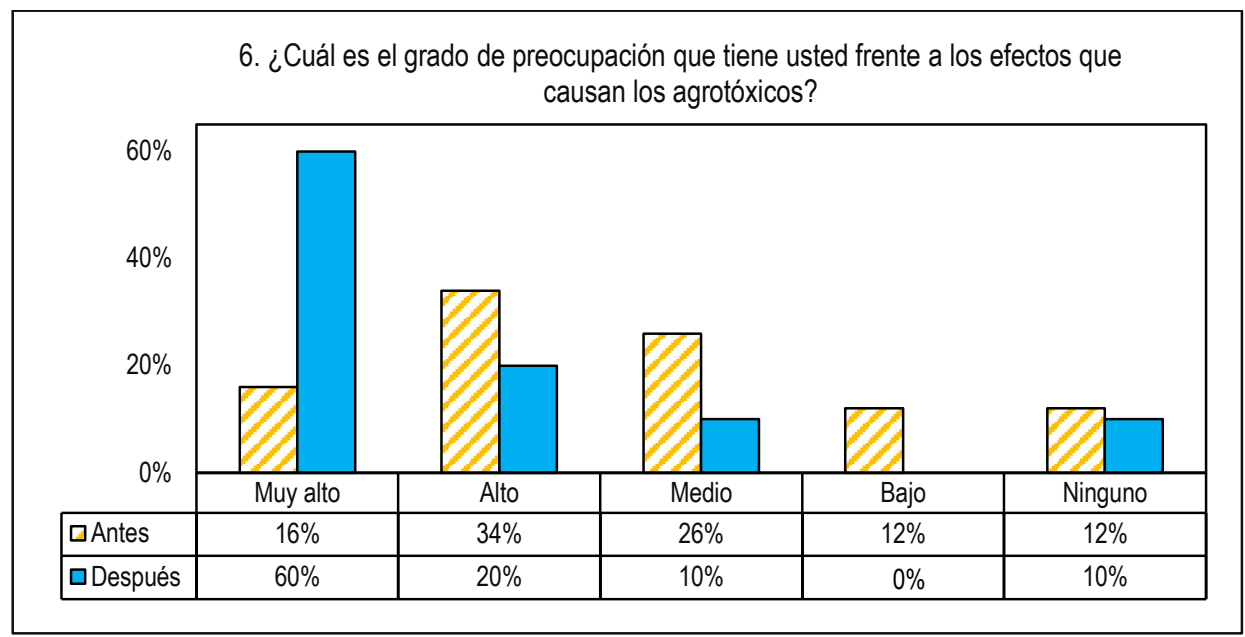

Fuente: Elaboración propia.

Actualmente, el $40 \%$ de los encuestados leen las etiquetas (5\% más que antes). Es importante señalar que el $30 \%$ de los moradores leen el etiquetado a veces; pues lo consideran innecesario (figura 8). García (2012) afirma que, en su gran mayoría, los agricultores no leen las indicaciones al utilizar agrotóxicos y, en caso de hacerlo, no aplican lo sugerido en las etiquetas, pues sus prácticas agrícolas se basan en los resultados que esperan obtener y para esto suelen pensar que, a mayor cantidad de agrotóxico, mejor será el efecto de estos.

Figura 8. Lectura del etiquetado en envases de agrotóxicos.

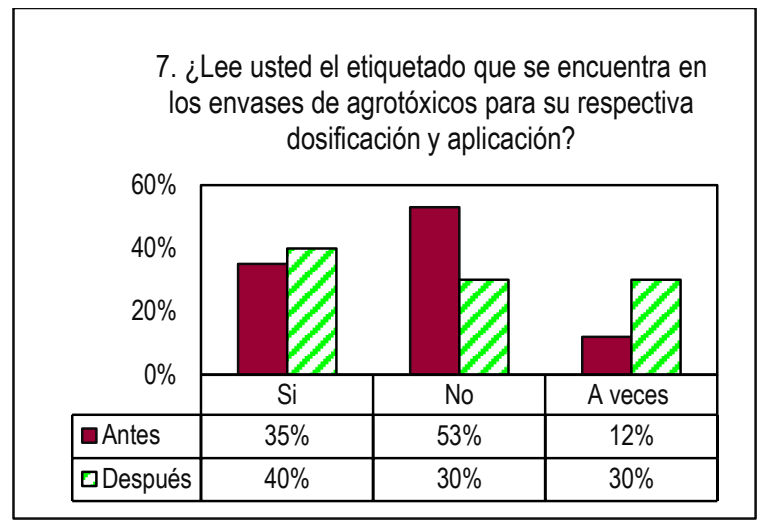

Fuente: Elaboración propia.

Respecto al uso de EPP, después de la aplicación de la estrategia un $72 \%$ de los encuestados afirmó usarlos; sin embargo, el $30 \%$ respondió que no lo utiliza. Varios agricultores mencionaron dicha negligencia porque esos equipos les causan molestias al realizar la fumigación (figura 9). Elahi et al. (2019) encontraron que el uso de protección durante la aplicación de agrotóxicos reduce significativamente el riesgo a la salud humana. No obstante, García (2012) menciona que los agricultores consideran 
innecesario el uso de equipos de protección y que un $49 \%$ de ellos considera que bañarse luego de tener contacto con cualquier agrotóxico es la mejor protección que existe. Aunque se reconoce esta práctica como algo positivo, es transcendental que los agricultores conozcan sobre la importancia del uso de equipos de protección al manipular agrotóxicos.

Según la Federación Nacional de Cacaoteros (2011), en el etiquetado de los agrotóxicos se especifica qué equipo de protección individual debe usar para preparar y aplicar el producto, y cuáles son los pasos de primeros auxilios a seguir en caso de que ocurra un accidente; pues como indica Gavilanes (2014), la protección física de los usuarios, la reducción de la cantidad de producto usado en los cultivos, la minimización de daños al ambiente, el acopio y la disposición de envases, residuos y productos caducos son algunas de las medidas que se consideran prioritarias a nivel mundial.

Figura 9. Uso de EPP para la manipulación de agrotóxicos.

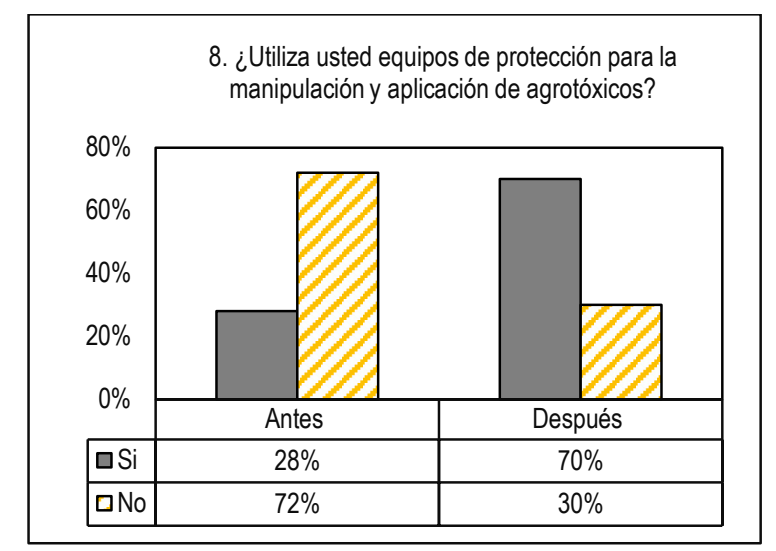

Fuente: Elaboración propia.

Respecto de la disposición final de envases de agrotóxicos (figura 10), el $80 \%$ de las personas afirma entregar los envases vacíos en un centro de acopio, también se pudo constatar que del $66 \%$ que los dejaba en el terreno, aún hay un $20 \%$ que lo hace; por otro lado, también se redujo la quema de estos (de $12 \%$ a $0 \%$ ).

Según la Federación Nacional de Cacaoteros (2011) y Hernández (2014), las actividades relacionadas con el manejo adecuado de agrotóxicos están reguladas por la legislación ambiental existente, y su descripción busca a través del conocimiento reducir el riesgo de exposición a estas sustancias. Por lo cual, Guzmán et al. (2016) recalcan que el riesgo a sufrir intoxicaciones agudas por agrotóxicos está relacionado a la ausencia de conocimientos técnicos. 
Figura 10. Disposición final de envases vacíos de agrotóxicos.

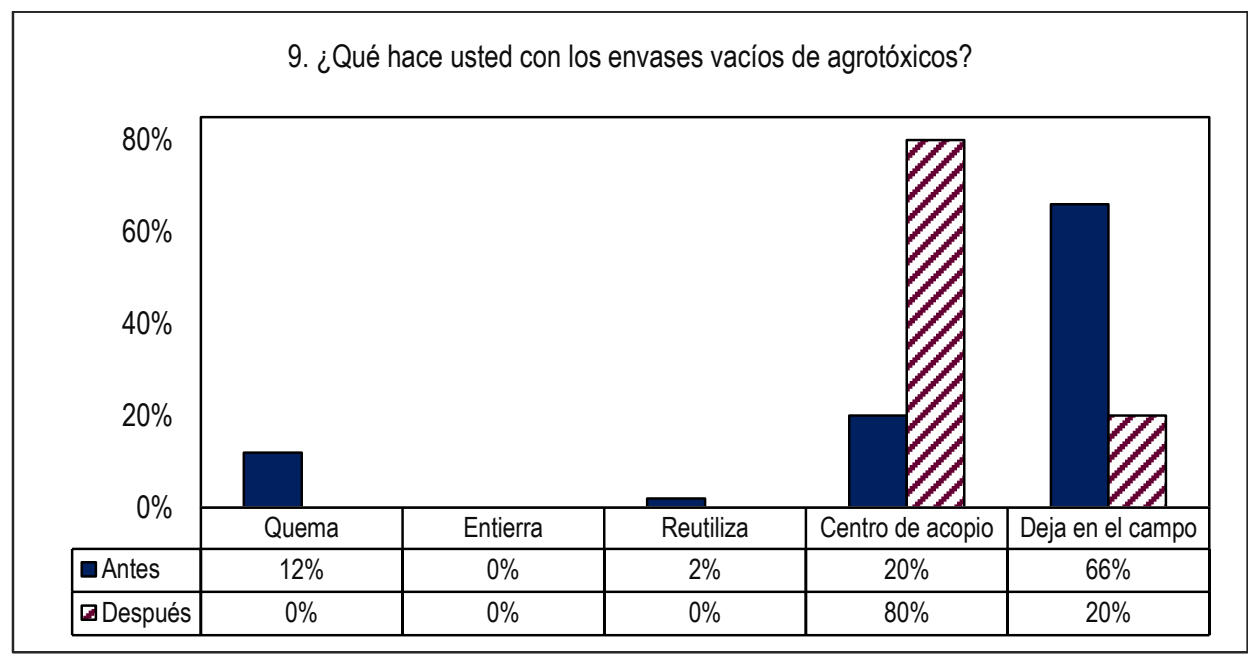

Fuente: Elaboración propia.

Es preciso mencionar que del Puerto et al. (2014) reiteran que el uso cotidiano de agrotóxicos contribuye a la crisis de la agricultura, lo que dificulta la preservación de los ecosistemas, los recursos naturales y afecta la salud de las comunidades rurales y de los consumidores urbanos. Por su parte, Ferreira et al. (2015) expresan que la búsqueda de la productividad a corto plazo por encima de la sustentabilidad ecológica, practicada en las últimas décadas, ha dejado un saldo a nivel mundial de contaminación y envenenamiento donde el pretendido remedio universal ha resultado ser peor que la enfermedad.

Los resultados obtenidos en esta investigación ponen en evidencia la necesidad de realizar intervenciones de educación ambiental en comunidades rurales, donde cada aporte de la academia puede significar la mejora en las condiciones de producción e incluso de la calidad de vida de las personas de este sector. Aunque este ha sido un primer acercamiento con la comunidad Bravos Grandes, se ha decido continuar su sensibilización con estos temas mediante la elaboración de materiales informativos virtuales, debido a la actual emergencia sanitaria. Este trabajo podría fomentar la divulgación de campañas de educación ambiental y promover un manejo adecuado de agrotóxicos en toda comunidad rural donde el uso de estas sustancias es cotidiano.

\section{CONCLUSIONES Y RECOMENDACIONES}

Las técnicas de investigación cualitativa revelaron que el uso de agrotóxicos es frecuente entre los agricultores de Bravos Grandes. En efecto, los resultados de esta investigación comprueban la necesidad de mejorar la disposición final de los envases de agrotóxicos y promover buenas prácticas ambientales en dicha comunidad, pues después de aplicar la estrategia de educación ambiental se evidenció un cambio de perspectiva acerca de su manejo, lo cual muestra la pertinencia de las intervenciones de este tipo en comunidades rurales. 
Gracias a la colaboración e interés de los moradores por participar en este programa, se logró aumentar el porcentaje de conocimiento sobre los agrotóxicos del $67 \%$ al $90 \%$. Así mismo, se mejoró la disposición final de envases vacíos, pues luego de aplicarse la estrategia, el $80 \%$ de los moradores utilizan el centro de acopio.
Dada la naturaleza de esta investigación resulta difícil indicar cuál fue el grado exacto de mejora en cuanto al manejo de agrotóxicos. No obstante, la sinergia lograda en el corto tiempo de capacitaciones constituye un aspecto clave a considerar en cuanto a las actividades de vinculación que cada institución de educación superior debe realizar.

\section{REFERENCIAS BIBLIOGRÁFICAS}

Bhandari, G. (2014). An Overview of Agrochemicals and Their Effects on Environment in Nepal. Applied Ecology and Environmental Sciences, 2(2), 66-73. http://doi.org/10.12691/ aees-2-2-5

Bravo, R., Zamora, Villafuerte, A., Peñarrieta, S., Santana, F., Zambrano, F., y Fimia, R. (2020). Diagnóstico de uso e impactos de plaguicidas en el cultivo de tomate (Solanumly copersicum I.) en la parroquia Riochico, cantón Portoviejo, provincia de Manabí, Ecuador. The Biologist, 18(1), 105-118. http://dx.doi.org/10.24039/rtb2020181476

Decnovk, A., de Troyer, N., Houbraken, M., Dominguez, L., Nolivos, I., Van, W., Eurie, M.A., Spanoghe, P. \& Goethals, P. (2019). Distribution of agricultural pesticides in the freshwater environment of the Guayas river basin (Ecuador). Science of the Total Environment, 646, 996-1008. https://doi.org/10.1016/i.scitotenv.2018.07.185

del Puerto, A., Suárez, S., y Palacio, D. (2014). Efectos de los plaguicidas sobre el ambiente y la salud. Revista Cubana de Higiene y Epidemiología, 52(3), 372-387. https://bit. ly/3hamk3i

Elahi, E., Weijun, C., Zhang, H., \& Nazeer, M. (2019). Agricultural intensification and damages to human health in relation to agrochemicals: Application of artificial intelligence. Land Use Policy, 83, 461-474. https://doi.org/10.1016/i.landusepol.2019.02.023

Federación Nacional de Cacaoteros. (2011). Uso y manejo seguro de plaguicidas en el cultivo del cacao 2011. Ministerio de Agricultura y Desarrollo Rural-Fondo Nacional del Cacao. https://bit.ly/34n8WYf

Ferreira, F., da Silva, L.G., Rigotto, R.M., Friedrich, K., \& Campos, A. (Coord). (2015). Dossiê ABRASCO: Um alerta sobre os impactos dos agrotóxicos na saúde. Escola Politécnica de Saúde Joaquim Venâncio-Associação Brasileira de Saúde Coletiva. Editora Expressão Popular. https://bit.ly/3nyiez4 
García, A. (2012). Conocimiento y uso de medidas preventivas por los agricultores en el manejo de agroquímicos en la comunidad Mojanda Mirador, cantón Otavalo, periodo enero 2012 -octubre 2012 [Tesis de grado, Universidad Técnica del Norte]. Repositorio Institucional UTN. https://bit.Iy/2KxiKrA

Gavilanes, G. (2014). La acumulación de envases de plaguicidas y su incidencia en la contaminación ambiental en el cantón Quero [Tesis de grado, Universidad Técnica de Ambato]. Repositorio Institucional UTA. https://bit.ly/2WjWQLf

Guzmán, P., Guevara, R., Olguín, J., y Mancilla, O. (2016). Perspectiva campesina, intoxicaciones por plaguicidas y uso de agroquímicos. Idesia, 34(3), 67-78. http://dx.doi.org/10.4067/ $\underline{\text { S0718-34292016000300009 }}$

Hernández, E. (2014). Propuesta de un programa y centro de educación ambiental en la región de Coatepec Harinas, Estado de México [Tesis de grado, Universidad Autónoma del Estado de México]. Repositorio Institucional UAEM. https://bit.ly/37oACOI

Herrera, M. (2011). Fórmula para cálculo de la muestra de poblaciones finitas. Investigación en Pediatría. https://bit.ly/3h2kDsy

Hidalgo, A., Romero, P., y Martínez, C. (2016). Estrategia de intervención comunitaria ambiental aplicada a la comunidad rural La Reforma en la Isla de la Juventud. Revista Novedades en población, 12(24), 94-103. https://bit.ly/2WniWfM

Hyland, C., \& Laribi, O. (2017). Review of take-home pesticide exposure pathway in children living in agricultural areas. Environmental Research, 156, 559-570. http://doi.org/10.1016/i. envres.2017.04.017

Instituto Nacional de Estadística y Censos. (2016). Información Ambiental en la Agricultura 2016. https://bit.ly/3nx48qu

Mantecón, C. (2015). Environmental education resources in Cantabria for preschool education [Tesis de grado, Universidad de Cantabria]. Repositorio Institucional UC. https://bit. ly/3mrwyRF

Ministerio de Agricultura, Ganadería, Acuacultura y Pesca. (2016). La política agropecuaria ecuatoriana: hacia el desarrollo territorial rural sostenible 2015-2025 (I Parte). https:// bit.ly/37y2Bv4

Ministerio del Ambiente del Ecuador. (2018). Estrategia Nacional de Educación Ambiental para el Desarrollo Sostenible 2017-2030. https://bit.ly/2WsFSu5

Naranjo, A. (2017). La otra guerra: la situación de los plaguicidas en el Ecuador. Acción Ecológica. https://bit.ly/38gSkTI

Pacheco, R.M., y Barbona, E.I. (2017). Manual de uso seguro y responsable de agroquímicos en cultivos frutihortícolas. Ediciones INTA. https://bit.ly/2KEO800 
Rohr, J.R., Barrett, Ch.B., Civitello, D.J., Craft, M.E., Delius, B., DeLeo, G.A., Hudson, P.J., Jouanard, N., Nguyen, K.H., Ostfeld, R.S., Remais, J.V., Riveau, G., Sokolow, S.H., \& Tilman, D. (2019). Emerging human infectious diseases and the links to global food production. Nature Sustainability, 2, 445-456. https://doi.org/10.1038/s41893-019-0293-3

Rossetti, M.F., Stoker, C., \& Ramos, J.G. (2020). Agrochemicals and neurogenesis. Molecular and Cellular Endocrinology, 510. https://doi.org/10.1016/i.mce.2020.110820

Shattuck, A. (2019). Risky subjects: Embodiment and partial knowledges in the safe use of pesticide. Geoforum. https://doi.org/10.1016/i.geoforum.2019.04.029

Yanggen, D., Crissman, C.C. y Espinosa, P. (2003). Los plaguicidas: impactos en producción, salud y medio ambiente en Carchi, Ecuador. Centro Internacional de la Papa, Instituto Nacional Autónomo de Investigaciones Agropecuarias. Editorial Abya Yala. 